\title{
Recurrent Renal Leiomyosarcoma Mimicking a Colonic Submucosal Tumor: A Case Report
}

\author{
Yun Jeung Kim, Hee Seok Moon, Eaum Seok Lee, Jae Kyu Sung, Hyun Yong Jeong, Ji Yeon Kim ${ }^{1}$ \\ Dae Young Kang ${ }^{2}$ \\ Departments of Internal Medicine, ${ }^{1}$ General Surgery, ${ }^{2}$ Pathology, Chungnam National University Hospital, Daejeon, Korea
}

\begin{abstract}
A primary leiomyosarcoma of the kidney is a rare, but highly aggressive, neoplasm, accounting for only $0.1 \%$ of all invasive renal tumors. Local or systemic recurrence is common, but a leiomyosarcoma is difficult to diagnose preoperatively. We recently encountered an interesting case of an unusual recurrence of a renal leiomyosarcoma. A 57-year-old woman visited our hospital complaining of lower abdominal pain. Four years previously, she had undergone a left nephrectomy. She had a primary leiomyosarcoma of the kidney that had been misdiagnosed as a renal cell carcinoma. Colonoscopy revealed the presence of a lesion similar to a submucosal tumor in the descending colon. Postoperative pathologic examination confirmed that the mass was a recurrent leiomyosarcoma. We report this unusual case and present a review of the literature.
\end{abstract}

Keywords: Leiomyosarcoma; Submucosal tumor; Recurrence; Colon; Kidney

\section{INTRODUCTION}

A renal leiomyosarcoma is an uncommon neoplasm of the kidney, accounting for approximately $1 \%$ of all malignant renal tumors, and has a poor prognosis $[1,2]$. It usually remains asymptomatic until the tumor grows to a relatively large size. Its symptoms, such as nonspecific abdominal discomfort, dull pain, and oliguria, are common in other conditions; therefore, a renal leiomyosarcoma is difficult to diagnose preoperatively. In this case, the condition had been preoperatively misdiagnosed four years earlier as a renal cell carcinoma before it was diagnosed as a primary renal leiomyosarcoma. Furthermore, it took several months to diagnose a recurrent renal leiomyosarcoma in the same patient because of the ambiguous, nonspecific symptoms. She visited our clinic complaining of diffuse abdominal pain, and colonoscopy

Received: March 21, 2011 Accepted: September 6, 2011

Correspondence to: Hee Seok Moon, M.D.

Division of Gastroenterology, Department of Internal Medicine, Chungnam National University Hospital, 640 Daesa-dong, Jung-gu, Daejeon 301-721, Korea

Tel: +82-42-280-7163, Fax: +82-42-257-5753

E-mail:mhs1357@cnuh.co.kr

(C) 2011 The Korean Society of Coloproctology

This is an open-access article distributed under the terms of the Creative Commons Attribution NonCommercial License (http://creativecommons.org/licenses/by-nc/3.0) which permits unrestricted noncommercial use, distribution, and reproduction in any medium, provided the original work is properly cited. showed a submucosal tumor or an extrinsic mass involving the descending colon. There are no reported cases of a renal leiomyosarcoma invading the retroperitoneal colon and mimicking a submucosal lesion. We report this case and present a literature review.

\section{CASE REPORT}

A 57-year-old woman visited our gastroenterology department complaining of diffuse lower abdominal discomfort. She had undergone a left nephrectomy four years earlier. The preoperative diagnosis was a renal cell carcinoma, but she was subsequently diagnosed with a primary renal leiomyosarcoma on pathologic examination (Fig. 1). Regular follow-ups with a urologist and abdominal computed tomography (CT) showed no recurrence for three years. After another year, she returned to the hospital complaining of diffuse abdominal discomfort. At that time, she was taking medication for hypertension and diabetes mellitus.

The findings of a general physical examination were unremarkable, except for diffuse abdominal sensitivity. The laboratory test results were as follows: leukocyte count, $6,770 / \mathrm{mm}^{3}$; hemoglobin level, $12.0 \mathrm{~g} / \mathrm{dL}$; and platelet count, $276,000 / \mathrm{mm}^{3}$. The levels of aspartate aminotransferase (12 IU/L), alanine transaminase (10 IU/ $\mathrm{L})$, alkaline phosphatase (82 IU/L), and total bilirubin $(1.2 \mathrm{mg} / \mathrm{dL})$ were within the reference range.

Colonoscopy revealed a hemispheric lesion similar to a submucosal tumor in the descending colon, approximately $30 \mathrm{~cm}$ above 
the anal verge (Fig. 2). The mass was found to be hard and fixed. An abdominal CT scan showed two mass lesions invading the descending colon (Fig. 3). The larger lesion was located in the peritoneum, adjacent to the anterior portion of the descending colon, and the smaller lesion was located in the retroperitoneum, adjacent to the posterior portion of the descending colon. Positron emission tomography-computed tomography (PET-CT) was performed. PET-CT showed a large omental mass on the left side of the abdomen, with a small solid area of increased glucose metabolism and hypermetabolism (maximum standardized uptake value of 13.7), in the posteromedial portion of the mid-descending colon (Fig. 4).

A laparoscopic tumorectomy and segmental resection of the descending colon were performed. Examination of the gross speci-
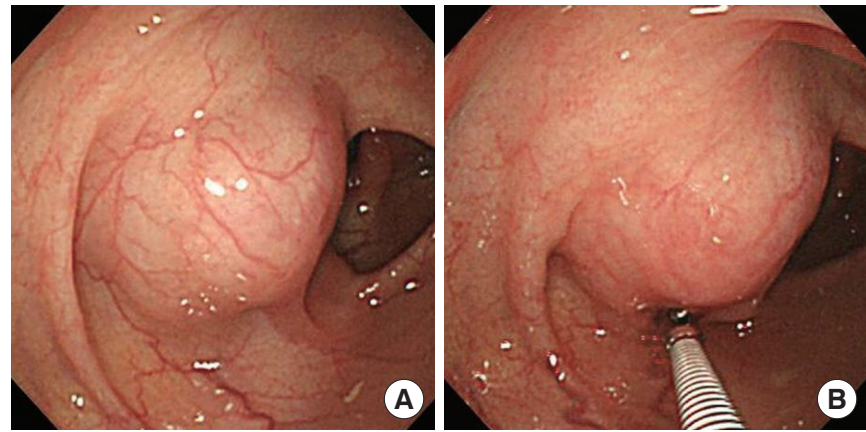

Fig. 2. Colonoscopic findings (A, B) Hemispheric lesion covered with normal appearing mucosa in the descending colon was hard and fixed, in compression by biopsy forcep.
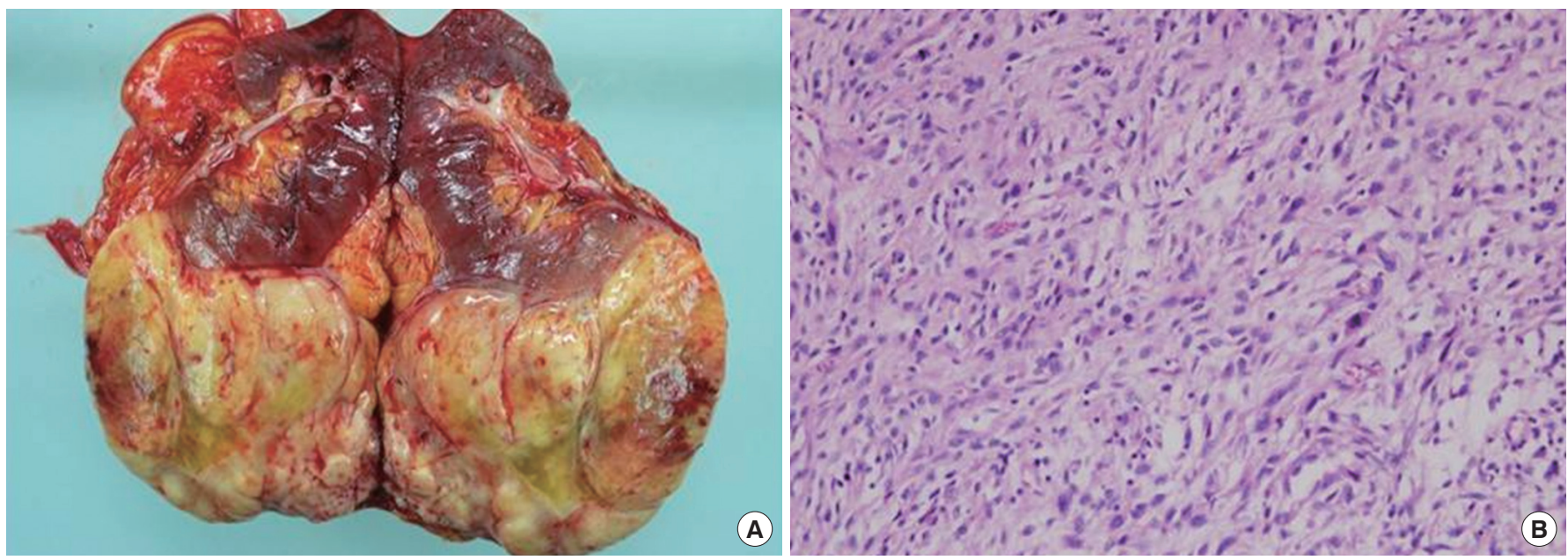

Fig. 1. Gross specimen and histopathologic findings of kidney. (A) Gross specimen shows a firm yellowish mass (approx. $10 \times 5.5 \mathrm{~cm})$. (B) Microscopic findings of renal leiomyosarcoma shows fascicles of spindle cells with elongated and blunt-ended nuclei $(\mathrm{H} \& \mathrm{E}, \times 200)$.
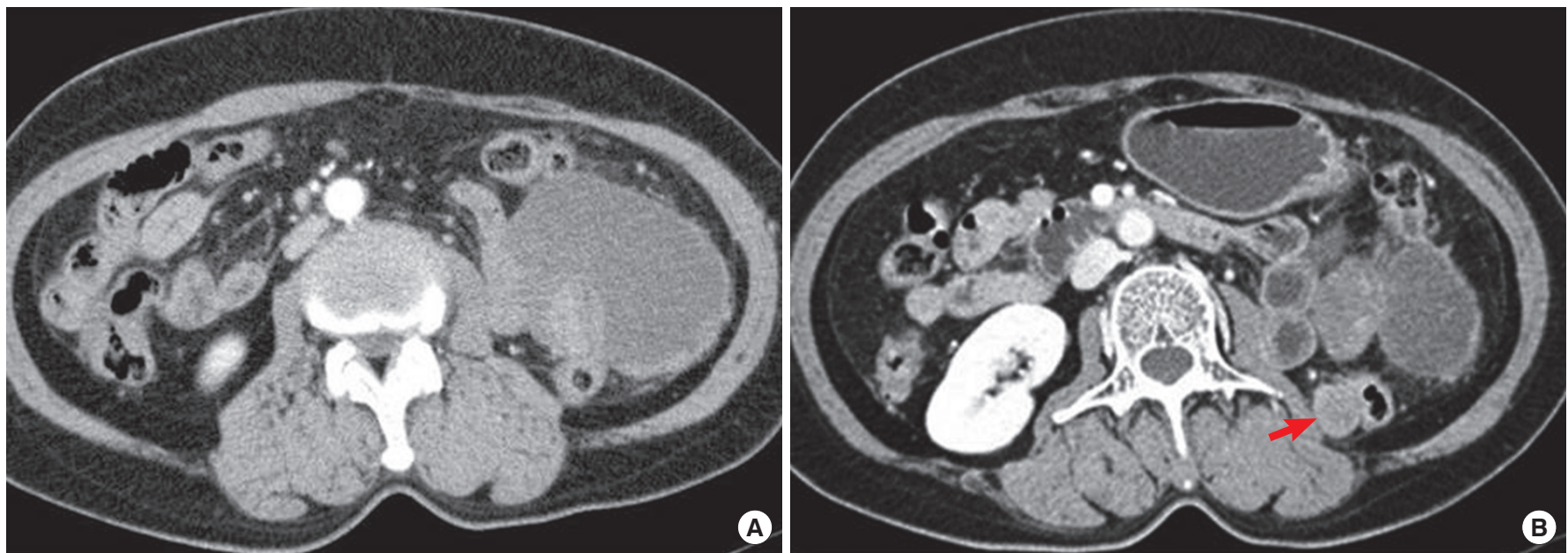

Fig. 3. Computed tomography imaging. (A) The large mass located in the peritoneum adjacent to the anterior portion of the descending colon; small portion of the mass showed increased enhancement. (B) Small mass situated in the retroperitoneum (red lined arrow) adjacent to the posterior portion of the descending colon. 
Journal of The Korean Society of Recurrent Renal Leiomyosarcoma Mimicking a Colonic Submucosal Tumor: A Case Report

Coloproctology Yun Jeung Kim, et al.

men revealed a firm white mass approximately $10 \times 9 \mathrm{~cm}$ and a $2 \times 2 \mathrm{~cm}$ mass invading the descending colon (Fig. 5).

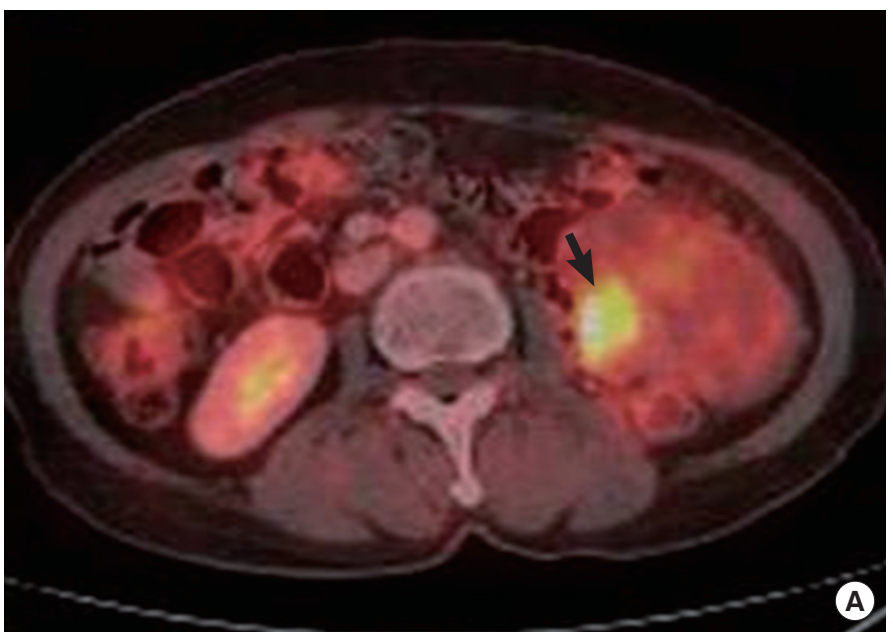

The final histopathologic diagnosis was a leiomyosarcoma. The number of mitoses was between 10 and 19 in each of 10 high-power

Fig. 4. Positron emission tomography-computed tomographic (PET-CT) imaging. (A) Large omental mass in the left abdomen and the solid portion of the mass, showed increased glucose metabolism (black lined arrow). (B) Hypermetabolic mass (maxSUV of 13.7) was detected in the posteromedial portion of the mid-descending colon (black dotted lined arrow).
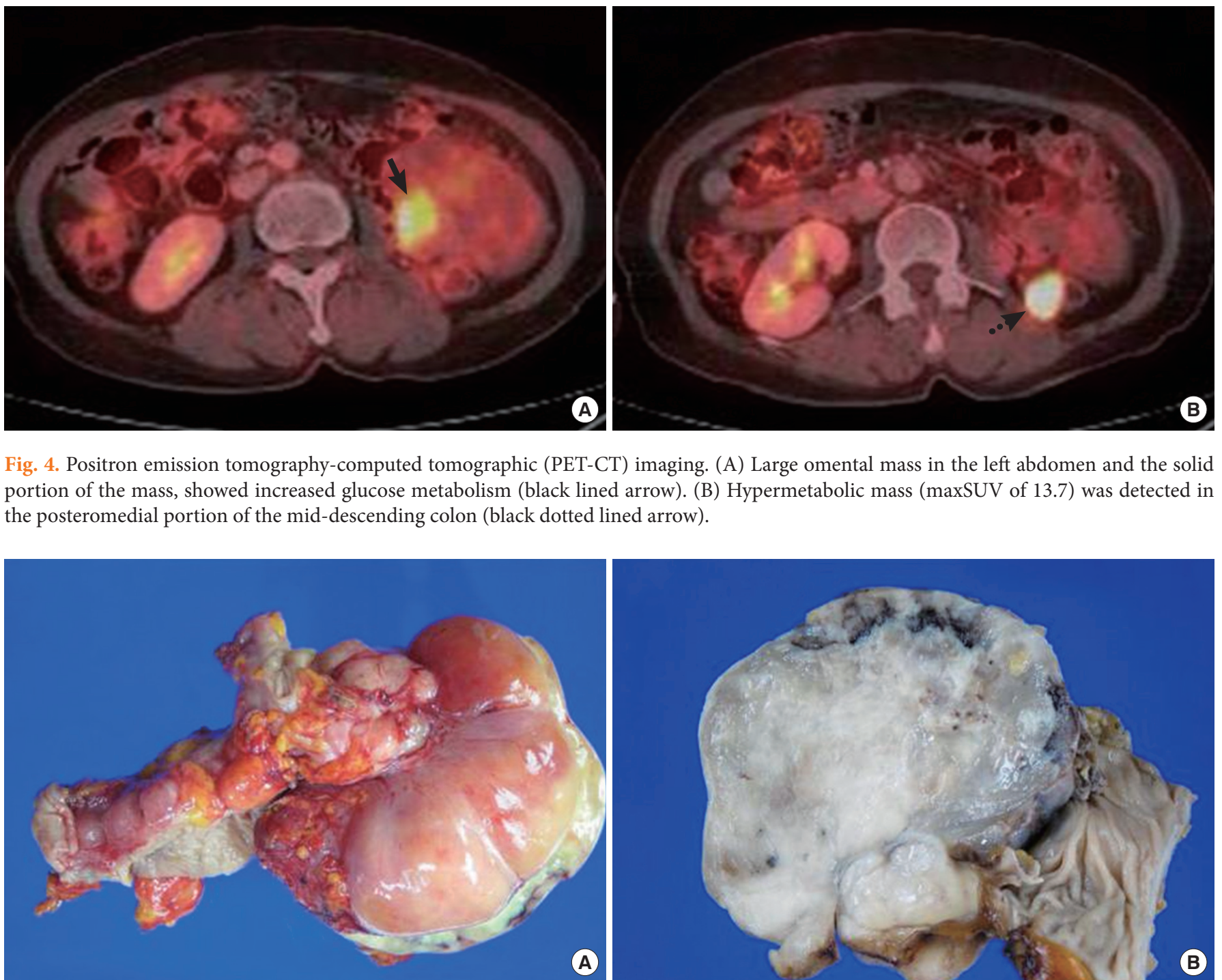

Fig. 5. Gross specimen. Gross specimen shows a firm white mass (approx. $10 \times 9.0 \mathrm{~cm}$ ) and a $2.0 \times 2.0 \mathrm{~cm}$-sized mass.
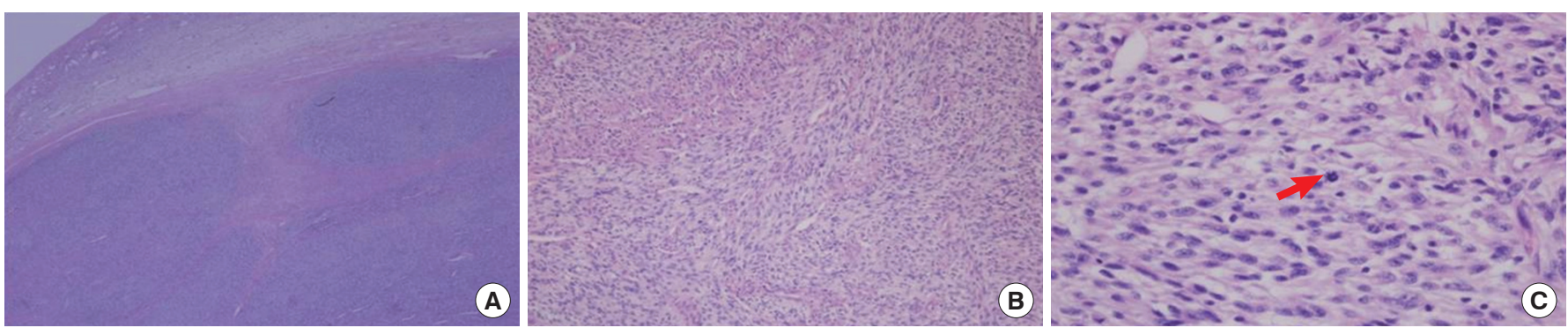

Fig. 6. Histopathologic findings Microscopic findings of recurrent leiomyosarcoma. (A) Solid tumor mass well demarcated by capsule with peripheral area $(\mathrm{H} \& \mathrm{E}, \times 20)$. (B) Fascicles of spindle cells with elongated and blunt-ended nuclei $(\mathrm{H} \& \mathrm{E}, \times 100)$. (C) Mitosis (red unbroken arrow) was observed $(\mathrm{H} \& \mathrm{E}, \times 400)$. 

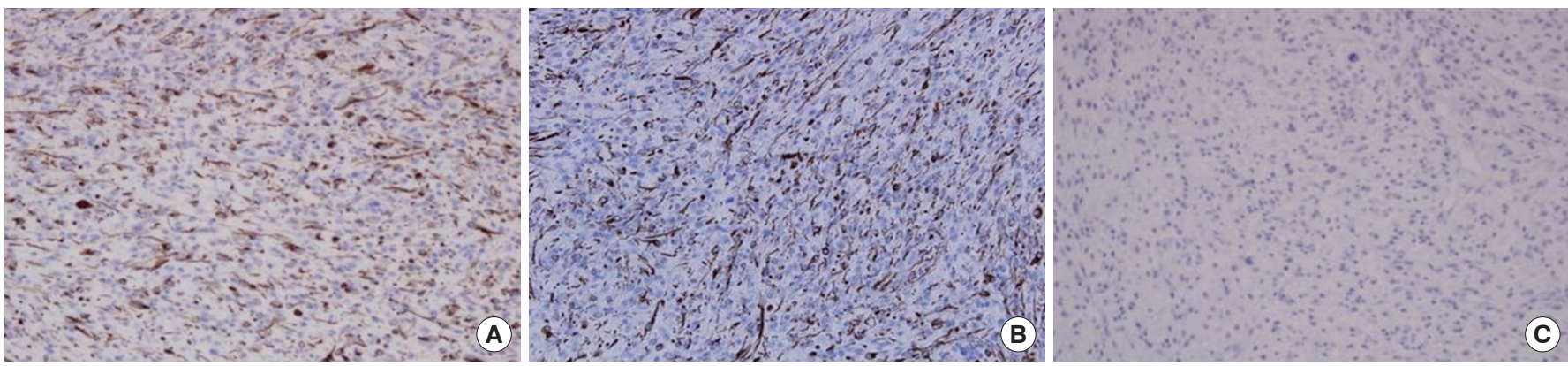

Fig. 7. Immunohistochemical staining results were positive in anti-smooth muscle actin (A), desmin (B), and negative in myosin (C).

fields; hence, the histologic grade was high. The microscopic examination ( $\times 20$ magnification) of a section stained with hematoxylin and eosin revealed a solid tumor mass, well demarcated by a capsule with a peripheral zone. Fascicles of spindle cells with elongated and blunt-ended nuclei were visible at $\times 100$ magnification (Fig. 6). Mitosis was identifiable at a magnification of $\times 400$ (Fig. 6C). Immunohistochemical staining showed positive results for smooth muscle actin and desmin (Fig. 7A, B) and a negative reaction for myosin (Fig. 7C). The patient then received two cycles of mesna, adriamycin, ifosfamide, and dacarbazine (MAID) chemotherapy at the oncology department.

\section{DISCUSSION}

Leiomyosarcomas are cancers of the smooth muscle cells, which can arise at any location. The uterus, the retroperitoneum, and the intra-abdominal cavity are common primary sites [3]. This tumor is most commonly seen in the fifth and the sixth decades of life, and the male-to-female ratio is 1:2 [4]. Leiomyosarcomas are characterized by a rapid growth rate and frequent local and systemic recurrences. High-grade leiomyosarcomas frequently metastasize, and the most commonly affected organ is the lung. Prognosis is poor, and the five-year survival rate is $29-36 \%$ [5]. In most reported cases, presentations are nonspecific and the results of physical examinations are normal. Radiologic features are nonspecific; therefore, it is difficult to diagnose this cancer preoperatively. A confirmed diagnosis is usually made only postoperatively, which results in delayed diagnosis. Primary renal leiomyosarcomas are sometimes misdiagnosed as renal cell carcinomas [6].

Four years earlier, our patient had also been diagnosed preoperatively as having a renal cell carcinoma based on radiologic examination, the physical examination findings having been nonspecific. The retroperitoneal recurrence of the cancer manifested only as mild diffuse abdominal discomfort. Leiomyosarcomas are distinguished from leiomyomas by cellular pleomorphism, an increased mitotic rate, and the presence of cellular necrosis. Furthermore, in this case, our pathologist conducted immunohistochemical staining, which is useful in the diagnosis of leiomyosarcoma. Immunohistochemical staining revealed positive findings for vimentin and smooth muscle actin and desmin. If a leiomyosarcoma specimen is found to have a pleomorphic component on immunohistochemical staining, it is difficult to distinguish it from a malignant fibrous histiocytoma. However, such heterologous differentiation was not observed in this case $[6,7]$.

The prognosis for a leiomyosarcoma is usually poor. A good prognosis can be expected only with complete surgical excision (the treatment of choice) [8-10]. Irradiation therapy and chemotherapy do not alter the course of the disease and are not successful in metastatic lesions [9]. A tumor size of less than $5 \mathrm{~cm}$, low histological grade, absence of lymph node metastasis, and radical excision of the mass are all associated with an improved prognosis [1]. To have ensured a better prognosis in this particular case, a radical nephrectomy should have been performed as the first operation. Unfortunately, as the patient had been thought to have a renal cell carcinoma in the preoperative state, she instead underwent a simple left nephrectomy.

Our case is notable and unusual because of the recurrence site and its features. The submucosal-like lesion was detected in the descending colon by using colonoscopy. A submucosal tumor is defined as an elevated or polypoid mass covered with normal mucosa. Several colorectal diseases, such as lipoma, leiomyosarcoma, lymphangioma, and carcinoid tumor, can be detected as lesions mimicking submucosal tumors. CT can help rule out extrinsic compression by abdominal masses. We also confirmed the lesion in the descending colon as being caused by extrinsic compression by performing a CT scan.

As observed in this case, recurrence of a leiomyosarcoma in the retroperitoneum needs to be considered in the differential diagnosis of submucosal tumors in the colon. There is no reported case of recurrence of a renal leiomyosarcoma in the colon. Thus, we report this unusual, but interesting, case of recurrence of a rare renal leiomyosarcoma.

\section{CONFLICT OF INTEREST}

No potential conflict of interest relevant to this article was reported. 


\section{REFERENCES}

1. Sharma D, Pradhan S, Aryya NC, Shukla VK. Leiomyosarcoma of kidney: a case report with long term result after radiotherapy and chemotherapy. Int Urol Nephrol 2007;39:397-400.

2. Moazzam M, Ather MH, Hussainy AS. Leiomyosarcoma presenting as a spontaneously ruptured renal tumor-case report. BMC Urol 2002;2:13.

3. Arnold LM 3rd, Burman SD, O-Yurvati AH. Diagnosis and management of primary pulmonary leiomyosarcoma. J Am Osteopath Assoc 2010;110:244-6.

4. Dhamne SA, Gadgil NM, Padmanabhan A. Leiomyosarcoma of the renal pelvis. Indian J Pathol Microbiol 2009;52:549-51.

5. Sharma B, Reddy M, Lee PC, Cortes G, Gumpeni R. An unusual clinical presentation of a rare renal tumour. Nephrol Dial Transplant 2010;25:1713-5.

6. Nicolas MM, Tamboli P, Gomez JA, Czerniak BA. Pleomorphic and dedifferentiated leiomyosarcoma: clinicopathologic and immunohistochemical study of 41 cases. Hum Pathol 2010;41:663-71.

7. Oda Y, Miyajima K, Kawaguchi K, Tamiya S, Oshiro Y, Hachitanda Y, et al. Pleomorphic leiomyosarcoma: clinicopathologic and immunohistochemical study with special emphasis on its distinction from ordinary leiomyosarcoma and malignant fibrous histiocytoma. Am J Surg Pathol 2001;25:1030-8.

8. Choudhury M, Singh SK, Pujani M, Pathania OP. A case of leiomyosarcoma of kidney clinically and radiologically misdiagnosed as renal cell carcinoma. Indian J Cancer 2009;46:241-3.

9. Miller JS, Zhou M, Brimo F, Guo CC, Epstein JI. Primary leiomyosarcoma of the kidney: a clinicopathologic study of 27 cases. Am J Surg Pathol 2010;34:238-42.

10. Demir A, Yazici CM, Eren F, Turkeri L. Case report: good prognosis in leiomyosarcoma of the kidney. Int Urol Nephrol 2007; 39:7-10. 